Cite this: Analyst, 2013, 138, 6246

Received 18th June 2013

Accepted 18th August 2013

DOI: 10.1039/c3an01204b

www.rsc.org/analyst

\section{Enhanced imaging of developed fingerprints using mass spectrometry imaging}

\author{
M. J. Bailey, ${ }^{\text {*a }}$ M. Ismail, ${ }^{\text {ab }}$ S. Bleay, ${ }^{c}$ N. Bright, ${ }^{a}$ M. Levin Elad, ${ }^{d}$ Y. Cohen, ${ }^{d}$ B. Geller, ${ }^{d}$ \\ D. Everson, ${ }^{a}$ C. Costa, ${ }^{a}$ R. P. Webb, ${ }^{\text {e J. F. Watts }}{ }^{f}$ and M. de Puit ${ }^{b}$
}

Latent fingermarks are invisible to the naked eye and normally require the application of a chemical developer followed by an optical imaging step in order to visualize the ridge detail. If the finger deposition is poor, or the fingermark is aged, it can sometimes be difficult to produce an image of sufficient quality for identification. In this work, we show for the first time how mass spectrometry imaging (in this case time-of-flight secondary ion mass spectrometry, ToFSIMS) can be used to enhance the quality of partially recovered fingermarks. We show three examples of how chemical imaging can be used to obtain enhanced images of fingermarks deposited on aluminium foil, glass and the handle of a hand grenade compared with conventional development techniques.

\section{Introduction}

The analysis of fingermarks has proven to be a very useful method for individualization purposes in relation to criminal activities. A prelude to the process of individualization is the visualization of latent, or enhancement of partially visible, fingermarks. The field of expertise of visualization of fingermarks holds many challenges but the ultimate goal is delivering material with sufficient information for individualization purposes.

As most fingermarks on exhibits are latent, some form of application of a reagent for the visualization has to be applied before the evidential value becomes apparent in the first place. Serial treatment of exhibits for this purpose is well known and is used in practice on a day to day basis. The guidelines as published by CAST (formerly HOSDB) describe the preferred sequence of application in order to obtain the highest possible yield of

${ }^{a}$ University of Surrey, Department of Chemistry, Guildford, GU2 7XH, UK. E-mail: m.bailey@surrey.ac.uk

${ }^{b}$ Netherlands Forensic Institute, The Hague, The Netherlands

${ }^{c}$ Centre of Applied Sciences and Technology, St. Albans, UK

${ }^{d}$ Division of Identification and Forensic Science, Israel Police, Israel

${ }^{e}$ Ion Beam Centre, University of Surrey, Guildford, GU2 7XH, UK

${ }^{f}$ Surface Analysis Facility, University of Surrey, Guildford, GU2 7XH, UK fingermarks in casework. ${ }^{1}$ In all of these cases the detection limits of the naked eye play an important role in the determination of the evidential value of fingermarks. Selecting the fingermarks lies at the fundament of the individualization process.

The reason for submitting a fingermark to a sequence of treatments can be twofold. If, after the first step of the visualization process no marks of any evidential value were detected, a follow up treatment increases the chances of a higher yield of useable marks. Secondly, a mark that was identified after the first treatment as holding some evidential value, can hold more evidential information which can be exposed when further treatment more of the area deposited by the donor of that mark becomes visible.

Whilst the naked eye has clear limitations, we will show that chemical imaging techniques can add sensitivity.

Chemical imaging techniques include spectroscopy and mass spectrometry. Imaging mass spectrometry techniques can offer enhanced selectivity and sensitivity compared with spectroscopy and include desorption electrospray ionization (DESI), matrix assisted laser desorption ionization (MALDI) and time of flight secondary ion mass spectrometry (ToF-SIMS). Recent work has shown that each of these techniques can be used to provide chemical images of the compounds found in fingermarks. ${ }^{2-4}$ Of these techniques, ToF-SIMS has superior spatial resolution and has been shown to provide good sensitivity to an array of endogenous and exogenous compounds in fingerprints, being sensitive to both organic and inorganic substances. ${ }^{2,5-7}$ ToF-SIMS is less destructive to the sample than MALDI or DESI, removing only a few monolayers of material. This low consumption leaves marks practically untouched and available for further analysis by other forensic disciplines.

In this paper, we show for the first time how mass spectrometry imaging can be used as a step in the fingermark development process to enhance visualization where conventional developers do not provide satisfactory results. We show three scenarios in which fingermarks that are only partially developed using conventional methods can be enhanced using ToF-SIMS. 


\section{Materials and methods}

\section{Samples}

Three different types of substrates and corresponding development procedures were used to investigate the feasibility of using ToF-SIMS to enhance the visualization of partially developed fingermarks:

a. Aluminium foil. Ungroomed fingerprints from a single Dutch donor were deposited on aluminium foil (as available from any supermarket), with the same finger sequentially touching the substrate (a so-called depletion series). The samples were treated using cyanoacrylate fuming at the Netherlands Forensic Institute (NFI), following the standard procedure as described in the safe operating procedure accredited to the ISO 17025 standard at NFI. A partially developed fingermark that had been aged for approximately two weeks was chosen for ToF-SIMS analysis and was photographed prior to analysis. The fingermark was then imaged at the Home Office Centre for Applied Science and Technology, UK, using a Minolta Dynax 7D digital camera fitted with a $50 \mathrm{~mm}$ macro lens. The sample was illuminated with a blue Crimelite $82 \mathrm{~S}$ (Foster \& Freeman, Evesham, Worcestershire) outputting in the range 420-470 nm, with a Schott glass GG495 yellow filter fitted to the camera.

b. Hand-grenade handle. Ungroomed fingerprints were deposited by 50 male donors on the handles of 50 hand grenades with a smooth surface, which consisted of a galvanized steel substrate with an organic coating. The fingerprints were developed using cyanoacrylate followed by crystal violet (10 handles), vacuum metal deposition (VMD) (10 handles), small particle reagent (SPR) (10 handles) black magnetic powder (10 handles) and black Wetwop ${ }^{\circledR}$ (10 handles) by the Latent Fingerprint Laboratory of the Division of Investigation and Forensic Science, Israel Police. The handles of these hand grenades are known from casework to be challenging for fingerprint development. In a follow-up study, CAST wet powder formulation was used. The CAST wet powder formulation uses a precipitated magnetic iron oxide powder, whilst black Wetwop ${ }^{\circledR}$ is based on carbon.

c. Glass. Sebaceous-loaded fingerprints from a single (British) donor were deposited on seven glass microscope slides and submerged in soil collected from the grounds at the University of Surrey for seven days. The soil was a sandy soil and was collected during wet weather. In order to deposit reproducible fingerprints, measures were taken to control the chemistry and thickness of deposit, as detailed in previous studies. ${ }^{2}$ In particular, the fingerprint donor washed their hands and face prior to deposition of the fingerprint and was then instructed to place their hands in plastic bags for 20 minutes. The bags were then removed and the donor was instructed to touch their forehead and nose to collect sebaceous material. This was followed by rubbing the hands together to homogenise the fingerprint residue. To deposit the fingerprint, the donor was instructed to use a light contact pressure with the substrate. Any soil residue was blown from the samples using a nitrogen gun directed at an oblique angle to the sample. Six of the fingerprint samples were then developed using one of aluminium powder, black magnetic powder, black particle suspension, basic violet 3, cyanoacrylate fuming and vacuum metal deposition at the Home Office Centre for Applied Science and Technology, UK. The fingerprints were imaged in transmitted light, placing them directly on a light box and photographing with a Minolta Dynax 7D digital camera fitted with a $50 \mathrm{~mm}$ macro lens. The seventh sample was reserved for ToF-SIMS analysis. The same procedure was carried out for fingerprints submerged in sea water (still, and from from Eastbourne, UK) for seven days.

\section{ToF-SIMS analyses}

Analyses were carried out on an IonToF GmbH (Munster, Germany) ToF SIMS 5 spectrometer, using a $20 \mathrm{keV} \mathrm{Bi}_{3}{ }^{+}$primary ion beam delivering $0.20 \mathrm{pA}$ of current. Images were acquired at $128 \times$ 128 resolution in the MacroRaster mode of operation. The 128 resolution was selected as it provides good image resolution within an acceptable time frame, i.e., just over $2 \mathrm{~h}$ per image acquisition. Superior quality images can be acquired employing higher resolutions; however these require respectively longer acquisition times. Image data were acquired using 512 cycles per pixel point with 1 scan per pixel. A cycle time of $100 \mu$ s was employed.

\section{Ethics}

All fingerprint donors used in this study were volunteers, who gave their fingerprints on the understanding that the fingerprints would not be stored in a database or used for identification purposes.

\section{Results}

\section{Aluminum foil}

Fig. 1 presents an optical image of a fingermark that was selected for study. The red box shows the area of the fingermark that was selected for ToF-SIMS analysis. The fingermark was not fully recovered by the cyanoacrylate fuming process - the ridges presented as a series of dots rather than continuous lines.

Fig. 2 shows ion images from the cyanoacrylate fumed fingerprint on aluminium foil in Fig. 1. Positive ions corresponding to $\mathrm{K}(\mathrm{m} / \mathrm{z} 38.97)$ and ${ }^{41} \mathrm{~K}(\mathrm{~m} / \mathrm{z} 40.96)$ were found in the fingerprint ridges, together with a number of negative ion species, presumed to originate from the polycyanoacrylate. The ridge detail in the negative ion images is so clear that it is even possible to see the pore structure in the ridges. Such pore

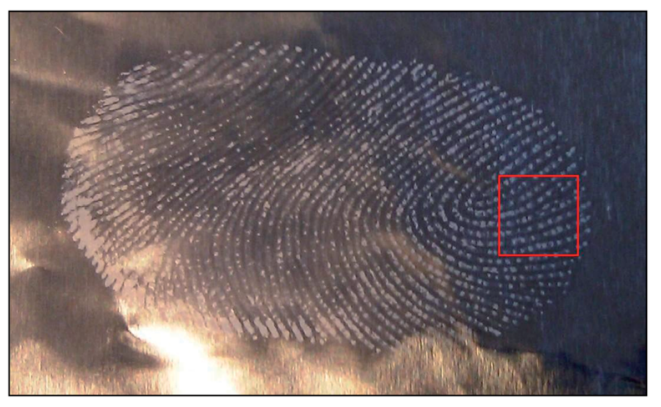

Fig. 1 Optical image of a fingermark deposited on aluminium foil and developed using cyanoacrylate fuming. The red box shows the $4 \times 4 \mathrm{~mm}^{2}$ area over which ToF-SIMS analysis was made. 

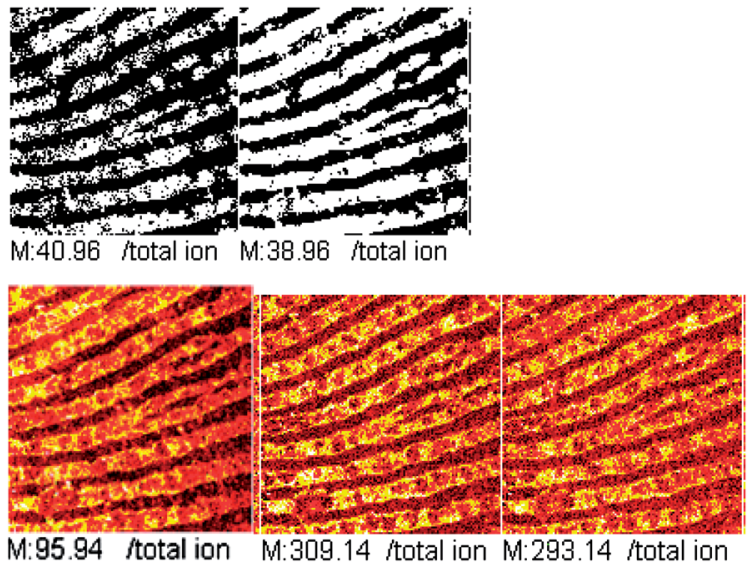

Fig. 2 ToF-SIMS images of a fingermark deposited on aluminium foil and developed using cyanoacrylate fuming shown in Fig. 1

structure is known as a level 3 fingerprint identifier and is not typically used by fingerprint examiners as it is rarely found. The ToF-SIMS images show that it is possible to produce continuous ridge images which cannot be produced with optical imaging.

To compare the performance of the technique with the best available imaging methods, the sample was analysed after ToFSIMS analysis using the previously described imaging set-up at the Home Office Centre for Applied Science and Technology. No visible change to the sample was observed following ToF-SIMS analysis, as shown in Fig. 3.

It is a noteworthy aside that in these samples only a few ions produced clear ridge detail, in contrast to previous studies. ${ }^{2,12}$ This is likely to be due to the fact that the cyanoacrylate monomer may have formed a layer over the entire sample and therefore species from the fingerprint cannot be detected by ToF-SIMS, which is only sensitive to the surface layers.

\section{Hand grenade handle}

Fig. 4 shows optical images of fingerprints aged for 24 hours and then developed using CAST formulation of wet powder and Wetwop ${ }^{\circledR}$. After 24 hours, no ridge detail could be developed using the conventional reagents: cyanoacrylate and crystal violet, VMD and SPR. Ridge detail was developed by Wetwop ${ }^{\circledR}$. However, after 24 hours no ridge detail could be developed even by Wetwop®. In the follow-up study, some ridge detail could be

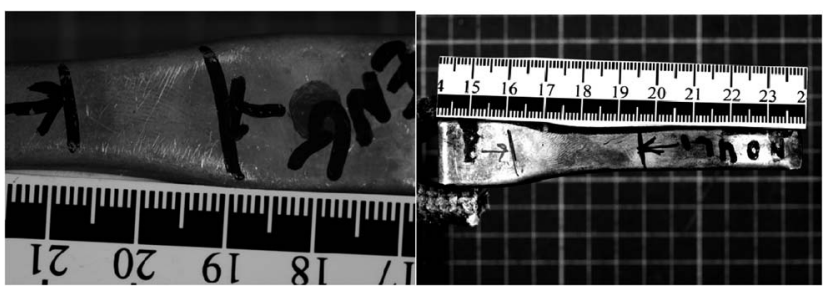

Fig. 4 Fingermark developed by (a) CAST formulation of wet powder after 4 days and (b) Wetwop® after 24 hours.

developed using CAST formulation after 4 days, but the ridge detail was insufficient, as shown in Fig. 4.

A $2 \times 2 \mathrm{~mm}^{2}$ sample was taken from the hand grenade handle and a fingerprint deposited on the handle. The fingerprint was imaged using ToF-SIMS 24 hours after deposition and 48 hours after deposition, as shown in Fig. 5a and b. $\mathrm{Na}^{+}(\mathrm{m} / \mathrm{z}$ 22.99) shows the location of the fingerprint on the sample and
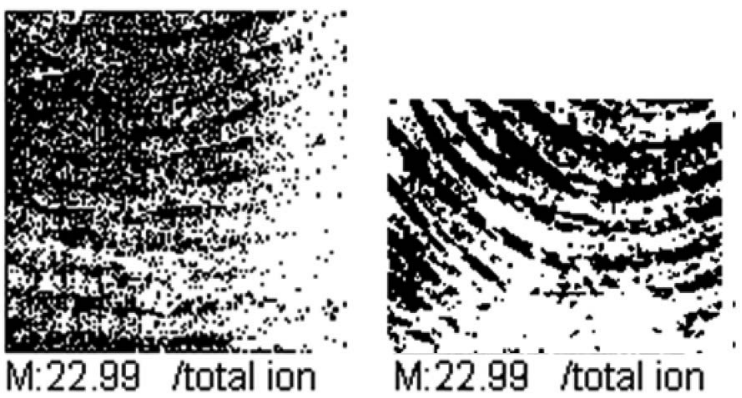

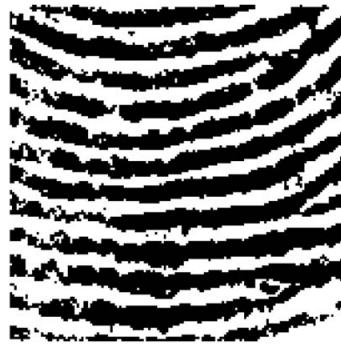

M:43.02/total ion

(a)

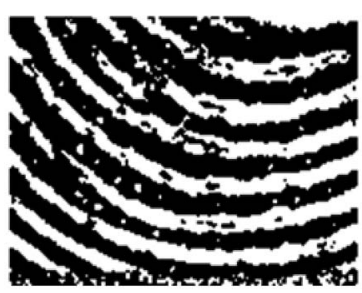

M:43.U2 /total Ion
Fig. 5 Images of $\mathrm{m} / \mathrm{z} 22.99$ (assigned to $\mathrm{Na}^{+}$) and $\mathrm{m} / \mathrm{z} 43.02$ (a fragment of PDMS) from a fingerprint deposited on a hand grenade handle (a) 24 hours and (b) 48 hours after deposition.

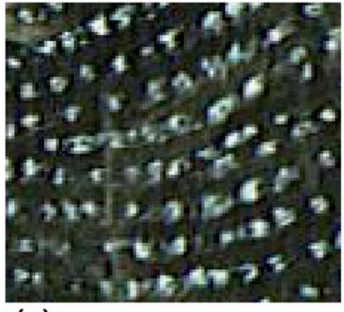

(a)

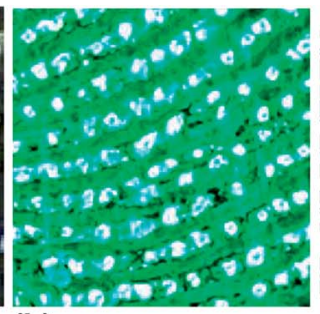

(b)

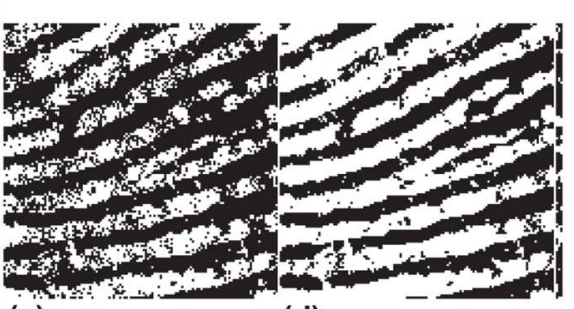

(d)

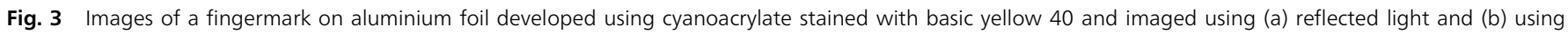
fluorescence examination compared with ToF-SIMS images (c), (d) from m/z 40.96 and 38.96 respectively, of the same area. 

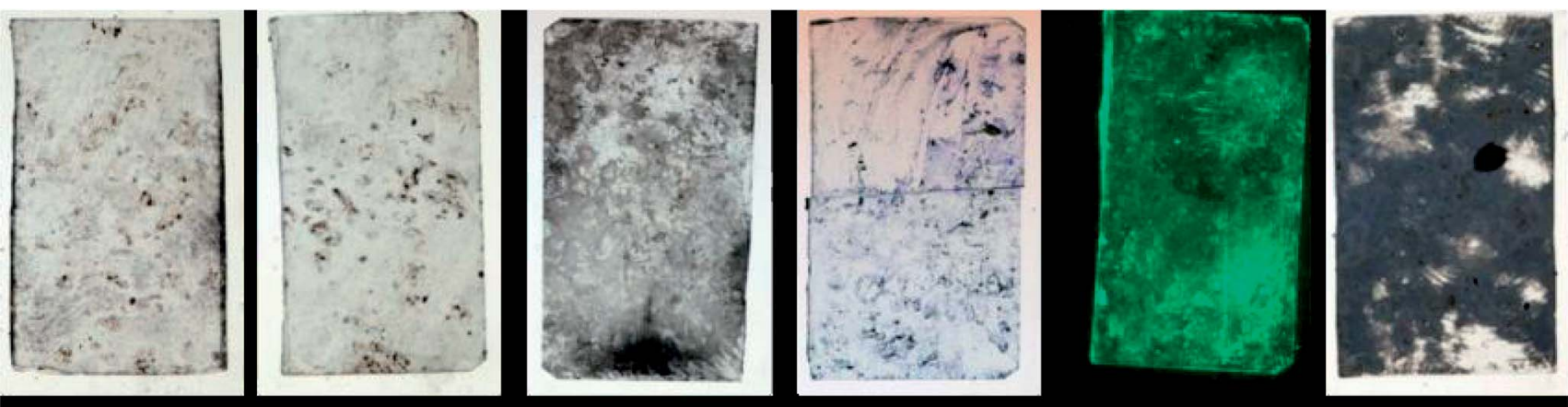

Fig. 6 Images of fingerprints deposited on glass slides, submerged in soil for 7 days and developed with (a) aluminium powder, (b) black magnetic particles (c) CAST formulation black powder suspension (d) basic violet 3 (e) cyanoacrylate fuming and (f) vacuum metal deposition.

some ridge detail is visible even 48 hours after deposition. Also detected and providing good contrast are ions at $\mathrm{m} / \mathrm{z} 43.02$, which are inversely correlated with the fingerprint ridges. These ions are from polydimethylsiloxane (PDMS), a well-known surface contaminant in ToF-SIMS spectra. In the images in Fig. 5, the fingerprint is white in the map of $m / z 22.99$ and black in the map of $m / z 43.02$.

\section{Glass}

Images of fingerprints deposited on glass substrates, submerged in soil for 7 days and developed using a suite of conventional developers are presented in Fig. 6. Ridge detail was not observed in any of the developed samples. Fig. 7 shows

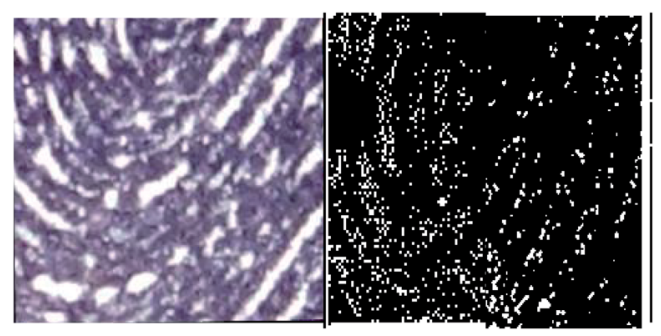

Fig. 7 Inked fingerprint (left) deposited on paper and corresponding ToF-SIMS image (right) from a sample exposed to soil for seven days. that by combining ion images of $m / z 57.09$ and 58.11 and subtracting the image of $m / z 43.01$ (from PDMS) some ridge detail is apparent. This is compared to a fingerprint from an inked finger deposited on paper to obtain a known exemplar. The ToF-SIMS image is significantly better than the results achieved by the conventional methods.

Fig. 8 shows the images of fingerprints immersed in seawater for seven days and recovered using a suite of conventional developers. Some ridge detail was recovered using vacuum metal deposition but the other developers failed to provide any ridge detail. Fig. 9 shows a ToF-SIMS image of a fingerprint exposed to the same conditions and compared with an inked fingerprint, again showing the potential of imaging mass

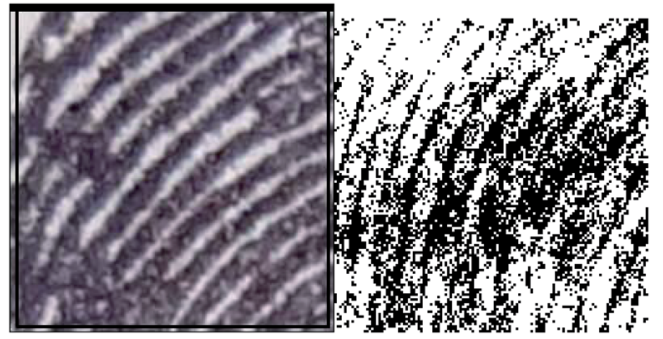

Fig. 9 Inked fingerprint (left) and corresponding composite ToF-SIMS image (right) from a fingermark exposed to soil for seven days.
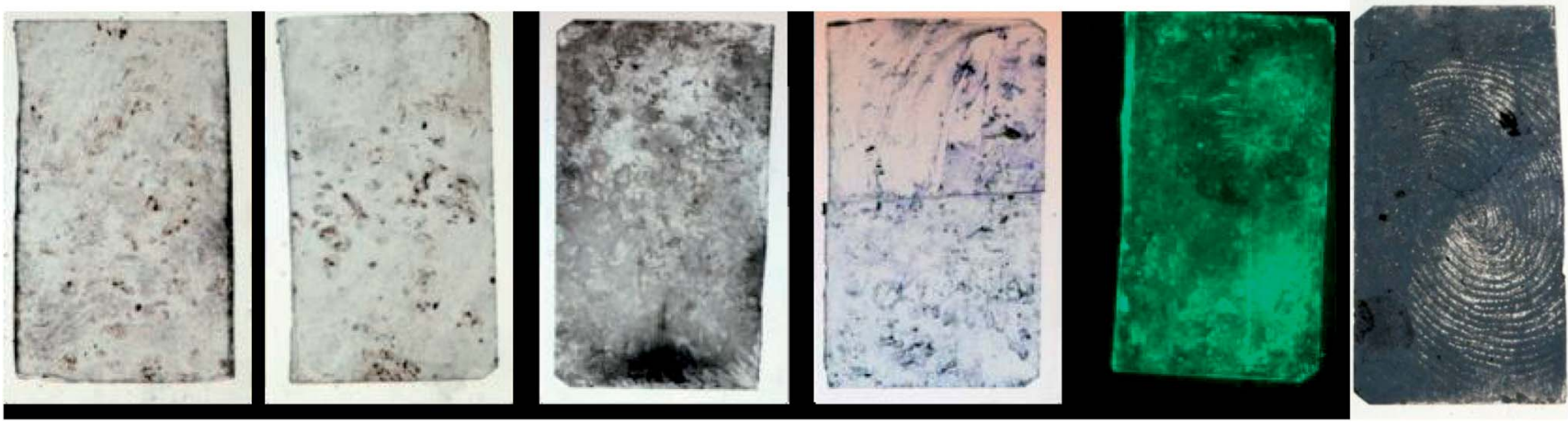

Fig. 8 Images of fingerprints deposited on glass slides, submerged in seawater for 7 days and developed with (a) aluminium powder, (b) black magnetic particles (c) CAST formulation black powder suspension (d) basic violet 3 (e) cyanoacrylate fuming and ( $f$ ) vacuum metal deposition. 
spectrometry to recover information that conventional developers miss.

\section{Discussion}

This work demonstrates for the first time the feasibility for using imaging mass spectrometry methods to image fingermarks that cannot be satisfactorily developed using conventional developers. The ToF-SIMS technique was chosen for this application due to its high spatial resolution and sensitivity as well as its quasi non-destructive nature. However a drawback of ToF-SIMS is the necessity to introduce samples into a vacuum chamber, which is known to cause changes to fingerprint chemistry, ${ }^{8}$ increase the time (and therefore cost) per analysis and impose limitations on the size of sample that can be analysed. At present, a sample of roughly $15 \times 20 \mathrm{~cm}^{2}$ could be accommodated in the vacuum chamber, but a large paper sample could take a couple of hours to outgas. Future studies should focus on the use of ambient pressure methods of imaging mass spectrometry. A technique which shows excellent promise for this application is MeV-SIMS, ${ }^{\mathbf{9}, \mathbf{1 0}}$ which also does not normally mark samples. The technique is currently under development but has recently been used to image samples under ambient conditions. With the construction of a suitable sample stage, it should be possible to acquire images of an entire fingerprint within a reasonable time frame ( $\sim 1$ hour).

Another limitation of this study is the limited number of donors that were used (each type of sample used fingerprints deposited by a different donor, so $n=3$ ) and the limited number of substrates that were tested. It is quite probable that any improvement provided by imaging mass spectrometry is donor and substrate dependent, as these factors are known to impact on fingerprint chemistry. ${ }^{\mathbf{1 1}}$ Additionally the compatibility of the technique with conventional development procedures is not yet known - although we have shown in this work that the technique works well after cyanoacrylate fuming.

\section{Conclusions}

We have shown that imaging mass spectrometry can be used to enhance visualization of latent fingerprints that are not satisfactorily developed using conventional developers. Although more work is needed in this area, we have shown the potential for ToF-SIMS to be used as a final step in the development process of fingerprints, where samples that have not been fully recovered using conventional reagents can be submitted for
ToF-SIMS. As we have shown here, the technique does not visibly change fingerprint samples, leaving them available for further testing by other forensic methods.

\section{Acknowledgements}

The authors would like to thank Steve Hinder from the Surrey Ion Beam Centre and Arian Van Asten from the Netherlands Forensic Institute for useful discussions.

\section{References}

1 Home Office Manual of Fingerprint Development Techniques, St Albans.

2 M. J. Bailey, N. J. Bright, R. S. Croxton, S. Francese, L. S. Fergusson, S. J. Hinder, S. Jickells, B. J. Jones, B. N. Jones, S. G. Kazarian, J. J. Ojeda, R. Webb, R. Wolstenholme and S. Bleay, Anal. Chem., 2012, 84(20), 8514-8523.

3 D. R. Ifa, N. E. Manicke, A. L. Dill and R. Graham Cooks, Science, 2008, 321(5890), 805.

4 R. Bradshaw, L. Ferguson, R. Wolstenholme, M. R. Clench and S. Francese, Anal. Chem., 2011, 83(14), 5585.

5 M. I. Szynkowska, K. Czerski, J. Rogowski, T. Paryjczak and A. Parczewski, Forensic Sci. Int., 2009, 184(1-3), e24.

6 M. I. Szynkowska, K. Czerski, J. Rogowski, T. Paryjczak, and A. Parczewski, Surf. Interface Anal. 42(5), 393.

7 N. J. Bright, R. Webb, S. J. Hinder, K. J. Kirkby, N. I. Ward, J. F. Watts, S. Bleay and M. J. Bailey, Anal. Chem., 2012, 84(9), 4083.

8 M. J. Bailey, N. Bright, et al., Forensic Sci. Int., 2013, 230(1-3), 81-86.

9 V. Palitsin, B. Jones and R. P. Webb, Presented at SIMS XVII, Toronto, September 2009, 2009; M. J. Bailey, B. N. Jones, S. Hinder, J. Watts, S. Bleay and R. P. Webb, Nuclear Instruments and Methods in Physics Research Section B: Beam Interactions with Materials and Atoms, vol. 268(11-12), 1929.

10 M. J. Bailey, B. N. Jones, S. Hinder, J. Watts, S. Bleay and R. P. Webb, Depth Profiling of fingerprint and ink signals by SIMS and MEV SIMS, Nuclear instruments and Methods in Physics Research Section B, vol. 268, iss. 11-12, pp. 19291932, Univ Cambridge, Cambridge, England.

11 R. S. Croxton, M. G. Baron, D. Butler, T. Kent and V. G. Sears, Forensic Sci. Int., 2010, 199(1-3), 93.

12 E. Sisco, L. Demoranville and G. Gillen, Forensic Sci. Int., 2013, 231(1-3), 263-269. 